\title{
eJRIEPS
}

Ejournal de la recherche sur l'intervention en éducation physique et sport

$17 \mid 2009$

Varia

\section{Le temps, le jeu et les sports collectifs. Le groupe « sports collectifs » du GRIAPS}

Didier Caty, Ching-Wei Chang, Paul Godbout, Jean-Francis Gréhaigne, Patrick Marle, Jean-Noël Meunier, Meher Rezig, Zeineb Zerai et Makram Zhigbi

\section{(2) OpenEdition}

\section{Journals}

Édition électronique

URL : http://journals.openedition.org/ejrieps/5547

DOI : $10.4000 /$ ejrieps. 5547

ISSN : 2105-0821

Éditeur

ELLIADD

\section{Référence électronique}

Didier Caty, Ching-Wei Chang, Paul Godbout, Jean-Francis Gréhaigne, Patrick Marle, Jean-Noël Meunier, Meher Rezig, Zeineb Zerai et Makram Zhigbi, « Le temps, le jeu et les sports collectifs. Le groupe « sports collectifs » du GRIAPS », eJRIEPS [En ligne], 17 | 2009, mis en ligne le 01 avril 2009, consulté le 18 mars 2021. URL : http://journals.openedition.org/ejrieps/5547 ; DOI : https://doi.org/ 10.4000/ejrieps. 5547

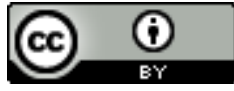

La revue eJRIEPS est mise à disposition selon les termes de la Creative Commons Attribution 4.0 International License. 
Le temps, le jeu et les sports collectifs

Le groupe « sports collectifs » du GRIAPS

(Didier Caty, Ching-Wei Chang, Paul Godbout, Jean-Francis Gréhaigne, Patrick Marle, Jean-Noël Meunier, Meher Rezig, Zeineb Zerai, Makram Zhigbi).

GRIAPS. IUFM de l'Université de Franche-Comté

\section{Résumé}

Cette contribution est une synthèse de l'état de la réflexion du groupe "sports collectifs " du Groupe de Recherche sur l'Intervention dans les Activités Physiques et Sportives concernant la notion de temps. Nous entendons revenir sur la place du temps dans l'analyse et la compréhension du jeu dans différents sports collectifs. Certes, le temps et l'espace constituent un système mais cette façon de penser les aspects temporels comme premiers devrait permettre de passer de "j'ai de la place " à "j'ai le temps de passer la balle » ou “j'ai le temps de passer avec le ballon ». Avec ce type de réflexion, les rapports de vitesse deviennent premiers, le démarquage et l'espace libre ne constituant alors que des conséquences des rapports de vitesse, donc du facteur temps entre les joueurs. Enfin, ce type d'analyse devrait permettre de passer d'une conception statique à une conception plus dynamique de la trame de transformation que constituent les rapports d'opposition dans une rencontre de sport collectif.

Parler du temps, c'est tout d'abord la résultante d'un apprentissage subjectif. En effet, à partir de cette expérience, nous sommes amenés à imaginer un espace et un temps qui nous sont propres et que nous devons aussi partager avec autrui. Ainsi, Bergson (1922) écrivait : "nous percevons le monde matériel, et cette perception nous paraît, à tort ou à raison, être à la fois en nous et hors de nous ". Pourtant, l'étude des faits, des évènements et du monde qui nous entourent, nécessite une description précise de l'espace et du temps, qui ne doit pas trop faire appel à la subjectivité et à l'implicite de l'observateur. La temporalité n'est-elle pas le mode d'être de l'homme ou de la femme, leur façon d'exister? Le temps n'est pas mesuré, il est vécu comme une durée. Si le vécu de la temporalité se réfère à la durée, c'est qu'il se joue dans l'intersection d'une durée longue et d'une durée courte, celle de l'événement, de l'action. Souvent, dans les sciences exactes, on représente le temps comme une droite avec un sens : la flèche du temps. On considère toujours un point sur cette droite, rarement le flux du temps, celui qui nous 
intéresse ici. Avec ces éléments, nous dirons que le temps renvoie à une durée occupée par un événement ou une action, voire à chacune des phases successives d'une action.

Dans l'enseignement des sports collectifs, on a souvent confondu plan horizontal, espace et temps. Toutefois, le caractère irréversible du temps devrait nous pousser à le traiter de manière radicalement différente de l'espace. Néanmoins, les notions spatiales dans les retours d'information et les propositions des professeurs d'EPS restent la référence quasi exclusive pour analyser et expliquer le jeu. Comment étudier et conceptualiser cette succession de formes fugaces ? Espace et temps sont-ils interchangeables ? De quels éléments disposons-nous pour mieux concevoir et comprendre la polysémie de ces deux termes ? C'est ce que nous allons tenter de faire dans ce texte.

\section{Quelques éléments dans la littérature}

Hughes (1980) insiste sur divers aspects tactiques pour créer des espaces libres et en particulier sur le jeu à une touche de balle. Pour l'auteur, ce type de jeu améliore la vision, la vitesse de décision, la vitesse de la balle et enfin le mouvement des attaquants. «Onetouch soccer encourages attacking players to move quickly into positions to help the player playing the ball. Furthermore, it encourages the player playing the ball to move quickly to a new position once the ball is played. [Le football à une touche de balle encourage les attaquants à se déplacer rapidement pour aider le porteur de balle. De plus, cela encourage le porteur de balle à se déplacer vite vers une autre position quand il a donné le ballon]» (p. 49). Comme on peut le noter, l'accent est déjà mis sur la vitesse et les déplacements.

La «vitesse de transmission du ballon » (VTB) est un indice chiffré mis au point par Dugrand (1985). Il est défini comme « le quotient du nombre de fois où un joueur reçoit le ballon (NR) par rapport au nombre de contact pied / ballon utilisé pour le transmettre $(\mathrm{NC}) \gg$,

soit VTB = nombre de balles reçues / nombre de contacts avec la balle.

Plus le quotient est proche de 1 , plus le joueur et/ou l'équipe jouent en une touche de balle.

Dugrand (1989) mentionne que « globalement la vitesse passe pour un argument majeur du jeu moderne ». On pourrait peut-être préciser ce point de vue en indiquant que c'est plutôt les variations de vitesse de la balle et des joueurs qui sont un argument majeur du football moderne. Cette petite précision apportée, il n'en reste pas moins que la VTB est un indice très intéressant au plan pédagogique même si elle n'est pas facile à relever et à exploiter. Lemoine et Jullien (2004), pour le jeu de haut niveau, complètent ce propos en 
affirmant que le jeu en déviation a été abordé par peu d'auteurs. Pourtant il est d'actualité : les réseaux défensifs des grandes équipes modernes sont si structurés et denses qu'ils ne permettent plus aux attaquants d'avoir des espaces d'évolution importants. Dans un contexte où il y a beaucoup moins de temps pour contrôler le ballon, sans être gêné d'une manière ou d'une autre par un adversaire, la déviation de la balle, associée à la vitesse de déplacement du joueur apparaît comme une réponse efficace pour d'une part faire circuler et conserver le ballon et d'autre part atteindre la cible adverse tout en tentant d'échapper aux marquages des défenseurs.

Gréhaigne (1989, 1992) met en évidence « des actions caractéristiques dans la phase offensive » en analysant le jeu en mouvement précédant un but. Pour cela, il réalise une étude approfondie des configurations de jeu qui se mettent en place juste avant la marque. II propose une modélisation des configurations momentanées du jeu qui provoquent un déséquilibre du système attaque / défense.

La rupture de l'état d'équilibre, la position du ballon et les espaces libres vont servir de base pour construire des outils d'observation afin d'analyser la transition entre deux configurations. Gréhaigne (1997) insiste, aussi, sur le rythme et les variations de vitesse dans le jeu: par exemple, une conduite de balle relativement lente pour engendrer un mouvement latéral chez l'adversaire puis accélération brutale lors de la réalisation d'une passe dans la profondeur. La perturbation ainsi créée change la physionomie du jeu. II est à noter également la notion de pression sur tel ou tel joueur, supposé faible, pour rompre l'équilibre des forces en présence dans les affrontements momentanés. On vise bien à créer un déséquilibre favorable pour aller marquer un but.

Marle, Pasteur \& Voland (1996) proposent dans leur progression d'acquérir les compétences et les savoirs en cinq cycles de formation. Leur grille d'analyse de chaque étape comprend une catégorie «temps » centrée autour de la rupture de vitesse et du changement de rythme.

Après cette rapide revue de la littérature, nous envisageons, dans ce texte, de montrer que les aspects temporels sont marquants dans l'étude des sports collectifs. Rien ne peut être fondamentalement compris dans le jeu si l'on ne passe pas d'un référentiel spatial à un référentiel temporel pour traiter les informations. On différencie ainsi les données tirées d'une prise d'information :

- de type séquentiel à partir de la prise en compte d'une succession de photographies instantanées ;

- de type continu à partir d'une analyse permanente que l'on stabilise de temps en temps. 
Ainsi, l'analyse des transitions entre les configurations relève soit d'un pari incertain soit d'une prévision cohérente d'évolution au temps $t+1$ ou $t+2$.

Pourquoi avoir choisi d'insister sur les aspects temporels des configurations du jeu ? Ils nous semblent fondamentaux pour une juste appréhension des décisions en jeu. En effet, il faut bien comprendre que l'on peut proposer la même tâche à des élèves débrouillés (de cours moyen par exemple) et à des professionnels en jouant uniquement sur la variable «temps ». Extérieurement la situation peut sembler la même, mais les exigences et la vitesse pour apporter une réponse efficace ne sont pas du tout du même niveau.

\section{De la dynamique du jeu}

Nous l'avons déjà écrit (Gréhaigne, 1989), une rencontre de sport collectif constitue un milieu complexe. Sur le terrain, la simple répartition spatiale des joueurs à différents endroits entraîne, le plus souvent, une répartition non homogène des états d'énergie des joueurs impliqués dans la configuration. Une certaine forme de dispersion homogène, qui caractérise l'état d'équilibre vers lequel le système d'affrontement évolue constamment en football, correspond donc, le plus souvent, à une homogénéité de la distribution des joueurs en fonction des rapports de vitesse entre ceux-ci et leurs localisations.

\subsection{Complexion du jeu}

Parfois, un degré d'homogénéité dans l'évolution d'une configuration du jeu peut aussi se concevoir par une distribution des probabilités de présence de joueurs à certains points clés du terrain (Gréhaigne, Bouthier \& David, 1997). Une autre façon équivalente de représenter les choses consiste à définir les micro-états du système attaque / défense à partir des emplacements, des directions et des vitesses possibles de tous les joueurs constitutifs de la situation de jeu à deux instants donnés proches. Ici la difficulté consiste en l'illustration de la vitesse des joueurs. Une possibilité existe avec la définition pour chaque micro-état d'une distribution des joueurs sur le champ de jeu en fonction de trois paramètres: leurs positions, leurs orientations et leurs vitesses de déplacement. En référence, et par analogie aux concepts utilisés par Planck (1941) en physique, une telle distribution constitue ce que nous pourrions appeler une complexion du jeu. La figure 1 présente un exemple de la modélisation d'une "complexion » et de son évolution probable en fonction des déplacements et de la vitesse des joueurs et du ballon. Cette configuration dynamique temporairement stabilisée permet d'anticiper sur l'évolution du rapport de forces. 


\section{Trajectoire balle \\ Trajet joueur}

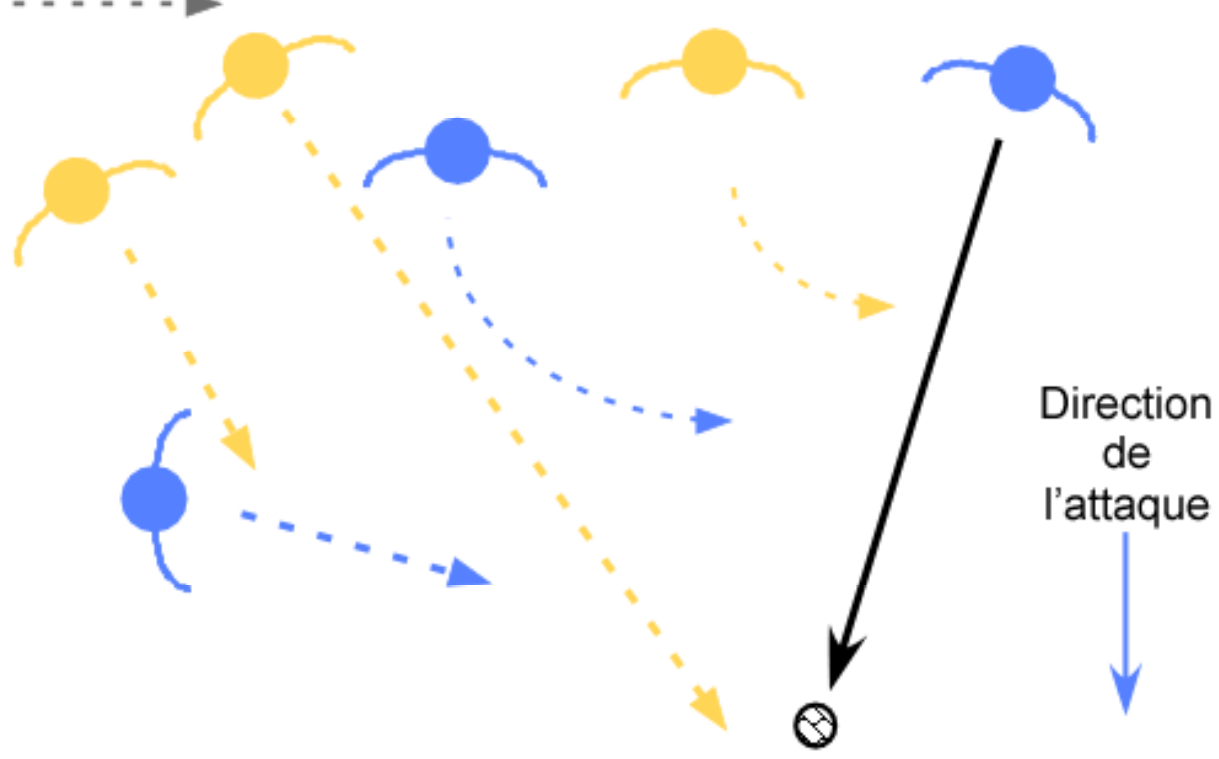

Figure 1. Exemple de représentation d'une complexion (entre T0 et $T+1$ ). Les flèches montrent la direction et la distance de déplacement en une seconde (indice de vitesse).

Dans notre cas, la défense possède une bonne chance de récupérer le ballon avec pourtant à l'origine une configuration spatiale assez défavorable (jeu dans l'espace libre avant par les «bleus » et la défense des «jaunes » étant plutôt à la poursuite). Les flèches représentent les trajets des joueurs en fonction de leurs déplacements, une sorte de transition stabilisée temporairement. Dans ce jeu en mouvement, les déplacements des attaquants ne se font pas tous dans la même direction, ce qui va modifier également le déplacement des défenseurs et faire évoluer la complexion du jeu.

Une autre façon de représenter une complexion du jeu consiste à modéliser le déplacement des joueurs en fonction de leurs emplacements de départ, leurs vitesses et leurs orientations. Pour représenter ces données dans le plan, nous proposons la notion de secteur d'action pour les attaquants et secteur d'intervention pour les défenseurs (voir la figure 2). Ces secteurs définissent spatialement les limites de l'action possible des différents joueurs par rapport aux trois paramètres cités plus haut (Gréhaigne \& Bouthier, 1994). 


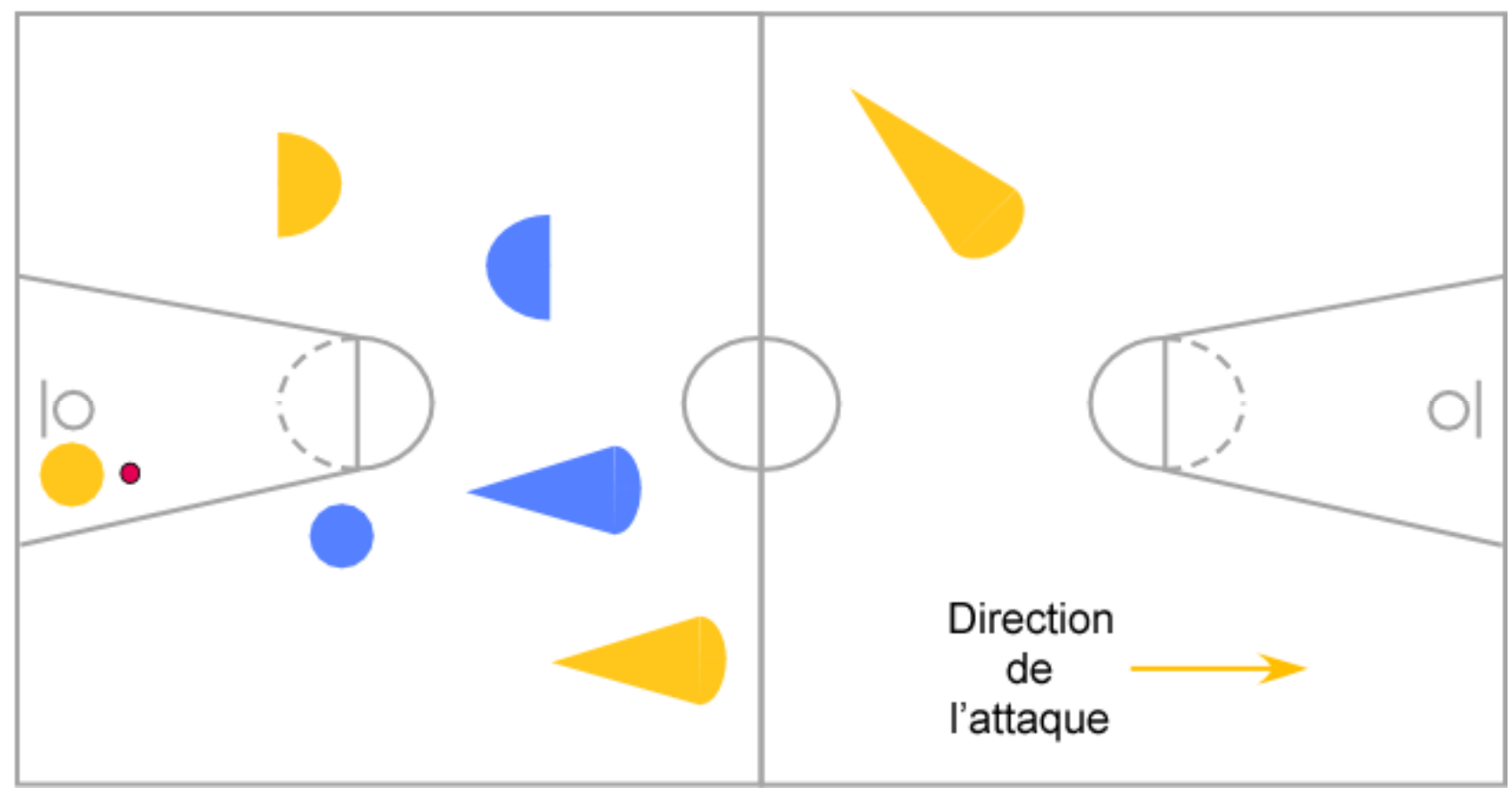

Figure 2. Secteur d'action pour les attaquants et secteur d'intervention pour les défenseurs dans une transition d'une seconde.

À un bon niveau, le désordre apparent, au sens physique du terme, désigne alors une homogénéité particulière autre que la simple répartition ou regroupement spatial des joueurs sur le terrain puisqu'il s'agit de distribution sur des niveaux énergétiques à partir de la construction par les sujets d'une transition temporairement stabilisée. Dans les situations d'opposition, ces interactions fondamentalement énergétiques (donc liées aux vitesses et aux accélérations / décélérations des joueurs en possession du ballon ou non) aboutissent à ce que des états spatialement non homogènes soient compensés et stabilisés par des distributions elles-mêmes hétérogènes mais apparaissant homogènes si l'on analyse les déplacements des joueurs. Cela veut dire que ces déplacements apparaîtraient homogènes à un observateur qui serait capable de décoder les niveaux énergétiques (les quantités de vitesse en interaction) tandis qu'une observation classique mettrait seulement en évidence des aspects hétérogènes mais structurés en rapport avec des positions et des formes géométriques. Les complexions du jeu possèdent en ellesmêmes une quantité de transformation(s) qui est à la fois restreinte en fonction des possibles de l'évolution continue du jeu, mais importante si l'on choisit une rupture en modifiant le mouvement en cours. Ainsi, l'objectif constant d'une équipe serait de conserver ou de récupérer son équilibre énergétique tout en tentant de déséquilibrer l'équipe adverse. En sport collectif, moins qu'ailleurs, il n'y a pas de progression linéaire ; on est toujours en présence d'états de déséquilibres et de recherches d'états d'équilibre à gérer en fonction de l'enjeu, du temps disponible, de ses connaissances et compétences motrices. Une des solutions à la crise de temps réside dans la capacité à anticiper sur 
l'évolution des configurations du jeu. Une bonne solution implique souvent de ne relever que quelques aspects distinctifs, un agencement partiel des éléments qui réunira toutes les interactions indispensables et elles seules pour analyser la configuration momentanée du jeu. Ochanine (1978) appelle « image opérative » la représentation chez le sujet de cet agencement partiel des éléments. C'est une image réduite aux éléments indispensables qui possède comme principales propriétés : l'adaptation à l'opposition, le laconisme, la plasticité et un caractère intentionnel.

Cette nouvelle façon d'envisager l'analyse du jeu nous renvoie, donc, à d'autres concepts pour comprendre ou prévoir l'évolution du jeu.

\section{Extension et compression du jeu}

Une vision dynamique de l'affrontement, comme par exemple le passage d'un état 1 « concentré » à un état 2 en « comète » (figure 3), entraîne la nécessité d'utiliser d'autres concepts pour rendre compte du jeu en mouvement. À cet effet, nous envisageons de tester les concepts d'espaces de jeu effectif en extension et / ou en compression afin de constater ce que pourrait amener cette autre façon d'envisager l'analyse du rapport de forces entre deux équipes.

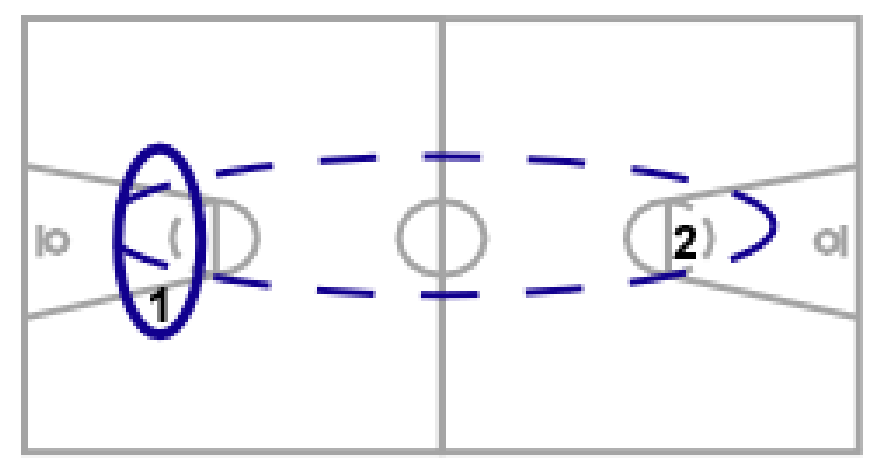

Figure 3. Transition d'un état 1 « concentré » à un état 2 en « extension ».

Les transitions et le jeu de transition renvoient souvent à des configurations où l'on a un peu de temps pour agir car la densité des joueurs est moins importante : l'attaque doit conserver son avance éventuelle et la défense doit revenir en barrage ou y rester (Gréhaigne, 2007, Ed.). Le recours au jeu long et / ou court transforme rapidement les configurations. Dès que le jeu long est possible, les configurations changent de forme et présentent le plus souvent un jeu en extension.

Le modèle en compression se retrouve souvent dans un jeu sur espace stabilisé avec une densité de joueurs importante. II présente des paradoxes ; il est à la fois le modèle des 
débutants et le modèle des experts, car il n'y a guère que les bons joueurs qui puissent être à l'aise dans des espaces restreints ou face à une défense de zone agressive. En ce qui concerne les débutants, la difficulté à lancer ou à bouger avec le ballon fait que le joueur en possession de la balle est statique et prisonnier de son orientation : il ne peut se déplacer que vers "son" avant. La pression de ses partenaires et celle de ses adversaires rendent difficile la poursuite du jeu ce qui entraîne, le plus souvent, un arrêt du ballon suivi par une perte de la balle.

3. 1. Des configurations.

Les figures 4 et 5 représentent une configuration du jeu en compression dans la zone d'attaque des noirs et sa modélisation sous forme d'espace de jeu effectif (EJE). Par définition l'espace de jeu effectif est une surface polygonale que l'on obtient en reliant entre eux les joueurs situés à la périphérie du jeu à un instant donné.

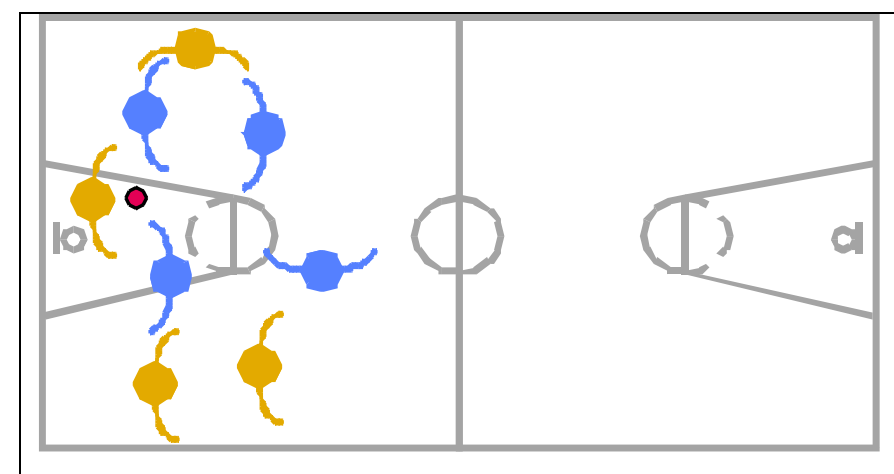

Figure 4. Une configuration du jeu.

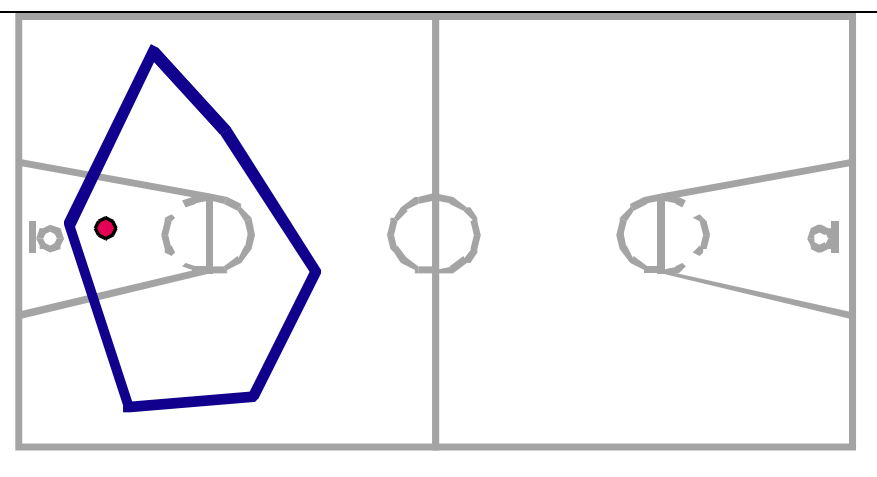

Figure 5. L'EJE de cette configuration.

Dans l'exemple (figures 4 et 5) le ballon est à l'arrière de l'espace de jeu effectif. La position du ballon par rapport à cet espace de jeu effectif permet de définir une défense en barrage ou une défense à la poursuite quand l'attaque est en avance sur le replacement défensif(Caty, Meunier \& Gréhaigne, 2007).

La figure 6 illustre l'EJE de l'attaque et de la défense pour cette même configuration.

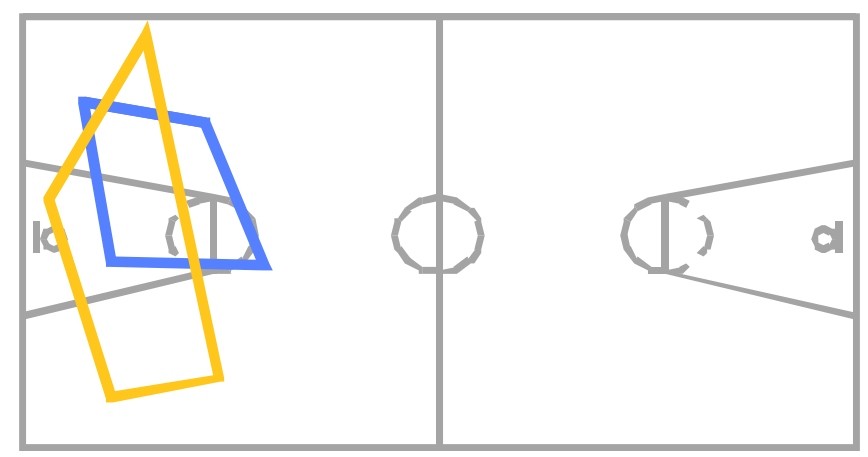

Figure 6. EJEA et EJED différenciés de cette même configuration. 
Les figures 7 et 8 représentent une configuration du jeu en extension dans la zone de transition des deux équipes et sa modélisation sous forme d'espace de jeu effectif (EJE).

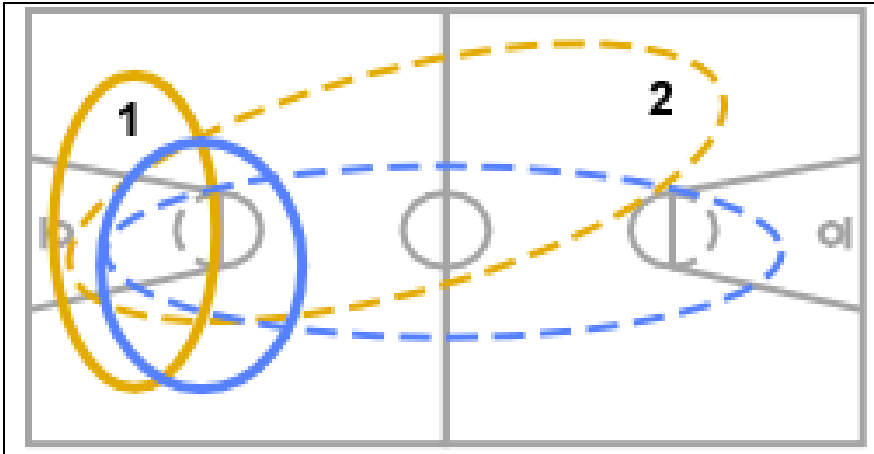

Figure 7. Une configuration du jeu en extension.

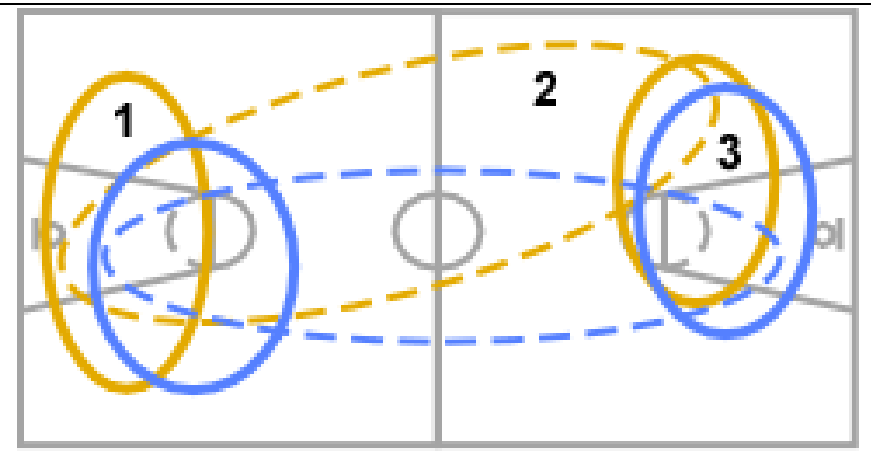

Figure 8. Retour à un jeu sur espace stabilisé.

Les phases de transition entre un jeu stabilisé dans la zone de défense et ce même jeu stabilisé dans la zone d'attaque sont très importantes à repérer car on va souvent de la compression vers l'extension et vice-versa. En effet, ces phases permettent d'anticiper sur les configurations momentanées à venir et ainsi de prendre les décisions adéquates. Ces différentes phases dépendent également du sport collectif considéré et de ses règles primaires et secondaires.

\section{2. Densité / concentration / évolution}

Dans une acception courante, la densité dans le jeu «mesurerait » la dispersion des joueurs sur une surface donnée. Ce serait donc, a priori, une valeur essentiellement spatiale et non numérique si l'on se réfère encore une fois à des états statiques. II existe une relation simple entre dispersion, densité et distance : plus la distance entre les joueurs augmente, plus la densité diminue et plus la dispersion augmente jusqu'à une limite où elle deviendrait constante. Les limites de ces phénomènes seraient constituées, sur un continuum, par une répartition uniforme de tous les acteurs sur l'aire de jeu, d'où une dispersion maximale de ceux-ci, ou un regroupement en un seul endroit sur le terrain, d'où une concentration maximale de tous les joueurs.

Dans la réalité, densité (variable qualitative) et dispersion / concentration (variables quantitatives) fonctionnent à l'intérieur de possibilités définies que sont les limites extérieures de l'aire de jeu utilisée. Dans une séquence de jeu, il peut exister, au même moment, une concentration dans certains endroits du terrain et une dispersion dans d'autres. Si l'on joue dans l'axe central, la dangerosité du mouvement en cours peut être appréciée en fonction du temps, des évènements du jeu et de la proximité de la cible ; il 
peut en résulter, par exemple, une concentration de joueurs dans l'axe but attaqué / but défendu et une dispersion importante à la périphérie du terrain.

Dans ce cadre, la concentration semble, aussi, une donnée utile à analyser pour bien comprendre l'évolution des rapports d'opposition dans un affrontement partiel et/ou dans le mouvement général. Dans le 1 contre 1 ou 1 contre plusieurs, pour un joueur particulier, une augmentation de la concentration des joueurs adverses peut permettre de mettre en évidence ses difficultés quand il doit jouer, sous pression temporelle, dans l'espace proche de ses adversaires. Ici, le temps pour effectuer un geste est souvent très réduit. Par contre, une certaine densité défensive peut être assurée avec une concentration minimale. Ainsi, un seul bon défenseur qui «flotte» intelligemment peut retarder plusieurs attaquants permettant le retour d'équipiers.

En conséquence, en sport collectif, la densité renverrait donc plutôt à un aspect qualitatif, la concentration / dispersion semblant plus liée à une approche quantitative en terme de nombre de joueurs. Compression / extension pour le mouvement et contraction / expansion à propos d'états statiques successifs (ou aspects temporels discontinus) semblent fonctionner en étroite symbiose pour décrypter et anticiper le mouvement dans le jeu et ainsi prendre des décisions judicieuses. Cela pourrait constituer un moyen d'expliciter les liens organiques entre les concepts de temps et d'espace ; néanmoins, cet ensemble de données renvoie bien, pour les phénomènes dynamiques continus, à des aspects temporels.

Dans le jeu, la couverture du terrain en défense, comme l'occupation de ce même terrain en attaque, est centrée autour de deux dimensions à occuper qui sont en partie contradictoires (Gréhaigne, Godbout \& Bouthier, 1999) : la largeur et la profondeur en relation avec l'étirement (dispersion) et/ou la concentration des joueurs de chaque équipe. On retrouve bien ici les idées de compression / extension même si l'on peut penser que c'est principalement la mobilité et les déplacements des joueurs qui assurent l'équilibre dans cette double tâche. II y a un dernier élément dont nous avons peu parlé dans ce texte : c'est le rythme du jeu et de ses variations. Nous allons aborder ce point maintenant.

\section{3. Rythme, variation du rythme et synchronisation}

Pour Teodorescu (1977), le rythme dans la défense consiste en des actions de défense plus ou moins nombreuses effectuées au cours d'une phase du jeu et par rapport à la durée de chaque action. On a pour habitude de dire qu'une défense agressive comporte normalement un grand nombre d'actions, tandis que la défense relativement passive en comporte un petit nombre. Néanmoins, l'importance du rythme est beaucoup plus grande dans l'attaque que dans la défense ; il dépend du nombre d'actions enchaînées, du tempo 
des actions individuelles et collectives dans l'attaque et de la durée de chacune d'entre elles. Suivant les actions de jeu, le rythme peut être rapide ou lent.

Les jeux sportifs collectifs modernes sont caractérisés par des rythmes rapides, soutenus également par l'utilisation délibérée de variations de rythme, afin de dérouter l'adversaire et de déjouer ses prévisions. Les variations du rythme renvoient souvent aussi à des variations de vitesse des joueurs, aux appels de balle, au passage d'un jeu en contournement à un jeu dans la profondeur, à une passe longue tendue déplaçant le jeu... Mais les variations du rythme renvoient également aux feintes, aux courses croisées, aux changements de direction, autant d'éléments qui permettent aussi de varier le rythme du jeu. En ce qui concerne un bon tempo, il nous semble que c'est jouer juste, au bon moment, en un mot au temps juste !

C'est la synchronisation des mouvements qui donne le moment opportun, le rythme interne à l'opération (action) et l'étendue de celle-ci dans les phases de jeu. La dimension temporelle contient deux éléments de durée imbriqués, l'un micro et l'autre macro (Fernandez, 2002). Une micro-durée règle les coordinations locales en termes de vitesse de déplacement, de débordement joueur à joueur par exemple. Une macro-durée correspond à la boucle « actions en projet / opérations / effets » dont les relations n'ont pas de limite définie, a priori, dans la stratégie mise en place. À terme, les deux temporalités se rejoignent, mais la conscience des phénomènes macroscopiques ainsi décrite semble toutefois plus difficile d'accès et d'apparition plus tardive. Néanmoins, il est possible que les ajustements dans un affrontement partiel ne soient pas toujours inscrits dans un projet stratégique global et que l'acte (tactique) individuel (Mahlo, 1969) puisse être localement désynchronisé, soit sans dommage pour la continuité de l'action ou des opérations, soit avec un bénéfice pour l'équipe adverse qui récupère alors le ballon. Une désynchronisation positive, dans un duel par exemple, consiste à rompre à son avantage l'équilibre des rapports d'opposition pour prendre de l'avance sur le replacement défensif. En conclusion de ce paragraphe, dans le rapport d'opposition, les unités contradictoires (jeu court / jeu long ; couverture / occupation ; compression / extension ; ordre / désordre ; arrêt / mouvement ; avance / retard ; lent / vite), avec les joueurs qui les exploitent ou les subissent, provoquent autant de situations où l'on domine et/ou on est dominé. Alors comment pouvons-nous modéliser ces aspects dialectiques du jeu ?

\section{Quelques considérations didactiques}

L'aspect temporel est aussi marquant dans l'étude des rapports d'opposition d'une rencontre car il sert de support à leur (s) fonctionnement (s) et surtout à leurs évolutions. 
On peut ajouter que finalement rien ne peut être fondamentalement compris en sport collectif si l'on ne passe pas d'un référentiel spatial à un référentiel temporel pour traiter des décisions en jeu. Mais, la reconnaissance et la construction du temps passe-t-elle nécessairement par des aspects spatiaux pour les élèves ? Est-ce qu'à certaines étapes de l'apprentissage le passage par un référentiel spatial est nécessaire pour construire le temps ? À l'heure actuelle, la réponse à ces questions demeure difficile car il reste toujours cette interrogation: comment enseigner ces aspects temporels ? Un premier élément nous est fourni par les jeux à effectif réduit. Le grand intérêt dans ce type de situation est de pouvoir donner du temps aux joueurs en diminuant la pression adverse et de jouer plus facilement, si on le désire, sur les variations de vitesse des joueurs et la vitesse de transmission du ballon à condition de proposer des situations où l'espace (la dimension du terrain) est en adéquation avec le nombre de joueurs. II convient, également, de bien distinguer l'activité du joueur en match et l'activité de l'élève en situation d'apprentissage. En situation de jeu usuel, le joueur se doit de mobiliser toutes ses ressources disponibles (perceptives, décisionnelles, émotionnelles, énergétiques, motrices...) dans des conditions d'urgence décisionnelle. L'essentiel de son activité consiste en une construction / reconstruction des configurations momentanées du jeu à partir d'indicateurs agencés en une image opérative qu'il prélève sur le jeu tels les placements et déplacements des joueurs, les trajectoires de la balle, les rapports de vitesse entre ces différents éléments, etc. En jeu réduit ou en situation d'apprentissage, l'activité du joueur est tout autre. D'une part, la contrainte temporelle peut être réduite, voire supprimée ; d'autre part, il s'agit pour celui-ci de construire des connaissances, des habiletés perceptives et décisionnelles ainsi que des compétences motrices par une succession d'essais en vue de transformer ses réponses habituelles. De ce fait, l'élève a la possibilité d'établir un projet avant d'agir, après une analyse a priori des éléments de la situation dans un débat d'idées. De même, il peut analyser ses réponses après l'action tant au niveau des effets qu'à celui des moyens utilisés. L'activité cognitive du joueur n'est donc pas de même nature selon qu'elle se situe dans la pratique sociale de référence, en jeu réduit ou encore en situation d'apprentissage. Recentrer les joueurs sur les données temporelles revient ensuite à les faire travailler dans des conditions où l'espace diminue et/ou le nombre de joueurs augmente, en un mot dans des conditions d'urgence temporelle de plus en plus prégnantes.

Enfin, une dernière série de données concerne le déroulement de ce jeu en mouvement et les rapports temporels entre défense et attaque. Dans le cas d'une défense à la poursuite, on constate généralement une tentative rapide de retour volontaire à l'équilibre, suivie du 
maintien de cet équilibre et enfin, quand la défense est stabilisée, on pense à la récupération du ballon. Ces éléments sont bien illustrés par le « recul-fuite » mais on les retrouve parfois dans le recul-frein quand un groupe de défenseurs en infériorité numérique tente de rester en barrage en « flottant » sans essayer vraiment de regagner la possession du ballon.

En conclusion, l'ambition de cette contribution était de montrer l'importance de penser « temps » quand on parle de didactique des sports collectifs, même à l'école. Certes, le temps et l'espace constituent un système mais cette façon de poser les aspects temporels comme premiers devrait permettre de passer de «j'ai de la place » à «j'ai le temps de passer la balle » ou « j'ai le temps de passer avec le ballon ». Avec cette façon de penser l'opposition, les rapports de vitesse entre les joueurs deviennent premiers, le démarquage et l'espace libre ne constituant alors que des conséquences des rapports de vitesse, donc du facteur temps entre les joueurs.

Enfin, ce type de réflexion et d'analyse devrait permettre de passer d'une conception statique à une conception plus dynamique de la trame de transformation que constituent les rapports d'opposition dans une rencontre de sport collectif. Cela oblige, sans doute, de passer d'une centration sur la circulation du ballon à une vision plus globale de la situation d'affrontement. Pour nous «Gagner du temps », c'est fondamentalement pour l'attaque prendre de l'avance sur le replacement défensif pour marquer et, pour la défense, c'est revenir en barrage entre le ballon et la cible pour éviter de prendre un but.

\section{Bibliographie}

Bergson, H. (1922). Durée et simultanéité. À propos de la théorie d'Einstein. Paris : P.U.F.

Caty, D., Meunier, J.N., \& Gréhaigne, J.F. (2007) Modélisations des attaques réussies pour progresser dans les sports collectifs en EPS. Spirales, 40, 105- 116.

Dugrand, M. (1985). Approches théoriques, expérimentale et clinique de l'enseignement du football. L'exemple au Sénégal. Thèse de troisième cycle, Université de Caen.

Dugrand, M. (1989). Le football : de la transparence à la complexité. Paris : PUF.

Fernandez, A. (2002). Rupture et prise d'avantage en sports collectifs, un modèle théorique. eJRIEPS, 2, 03-17.

Gréhaigne, J.F. (1989). Football de mouvement. Vers une approche systémique du jeu. Thèse (non publiée). Université de Bourgogne.

Gréhaigne, J.F. (1992). L'organisation du jeu en football. Paris : ACTIO. 
Gréhaigne, J.F. (1997). Modélisation du jeu de football et traitement didactique des jeux sportifs collectifs. Habilitation à diriger les recherches. Université de Paris-Sud Orsay.

Gréhaigne, J.F. (2007, Ed.). Configurations du jeu, débat d'idées et apprentissage des sports collectifs. Besançon : Presses de l'Université de Franche-Comté.

Gréhaigne, J.F., \& Bouthier, D. (1994). Analyse des évolutions entre deux configurations du jeu en football. Science et Motricité, 24, 44-52.

Gréhaigne, J.F., Bouthier, D., \& David, B. (1997). Dynamic systems analysis of the opponent relationships in the collective actions in soccer. Journal of Sports Sciences, 15, 137-149.

Gréhaigne, J.F., Godbout, P., \& Bouthier, D. (1999). The foundations of tactics and strategy in team sports. Journal of Teaching in Physical Education, 18, 159-174.

Hughes, C. (1980). The football association coaching book of soccer tactics and skills. London, England: Queen Anne Press

Lemoine, A., \& Jullien, H. (2004). Etude de la production d'Information dans le cadre de la transmission instantanée du ballon en football. eJRIEPS, 6, 47-55.

Mahlo, F. (1966). Acte tactique en jeu. Paris : Vigot.

Marle, P., Pasteur, R., \& Voland, C. (1996). Enseigner en sport collectif avec le football. Paris : AEEPS.

Ochanine, D. (1978). Le rôle des images opératives dans la régulation des activités de travail. Psychologie et Education, 2, 63-72.

Planck, M. (1941). Initiation à la physique. Paris : Flammarion.

Teodorescu, L. (1977). Théorie et méthodologie des jeux sportifs. Paris : Les Editeurs Français Réunis. 